\title{
Generation and comprehensive characterization of induced pluripotent stem cells for translational research
}

\author{
Chad C MacArthur ${ }^{\ddagger}{ }^{1}$, Suman Pradhan ${ }^{\ddagger}{ }^{1}$, Nichole Wetton $^{1}$, Aryan Zarrabi ${ }^{1}$, Carl Dargitz $^{1}$, \\ Mahalakshmi Sridharan ${ }^{1}$, Stephen Jackson ${ }^{1}$, Loni Pickle ${ }^{1}$ \& Uma Lakshmipathy*,1 \\ ${ }^{1}$ Cell Biology, Life Sciences Solutions, Thermo Fisher Scientific, Carlsbad, CA 92008, USA \\ *Author for correspondence: Tel.: +1 760268 7465; uma.lakshmipathy@thermofisher.com \\ ${ }^{\ddagger}$ Authors contributed equally
}

Induced pluripotent stem cells (iPSCs) hold immense potential in disease modeling, drug discovery and regenerative medicine. Despite advances in reprogramming methods, generation of clinical-grade iPSCs remains a challenge. Reported here is the first off-the-shelf reprogramming kit, CTS CytoTune-iPS 2.1, specifically designed for clinical and translational research. Workflow gaps were identified, and methods developed were used to consistently generate iPSC from multiple cell types. Resulting clones were subjected to characterization that included confirmation of pluripotency, preservation of genomic integrity and authentication of cell banks via an array of molecular methods including high resolution microarray and next-generation sequencing. Development of integrated xeno-free workflows combined with comprehensive characterization offers generation of high-quality iPSCs that are suited for clinical and translational research.

\section{Graphical abstract:}

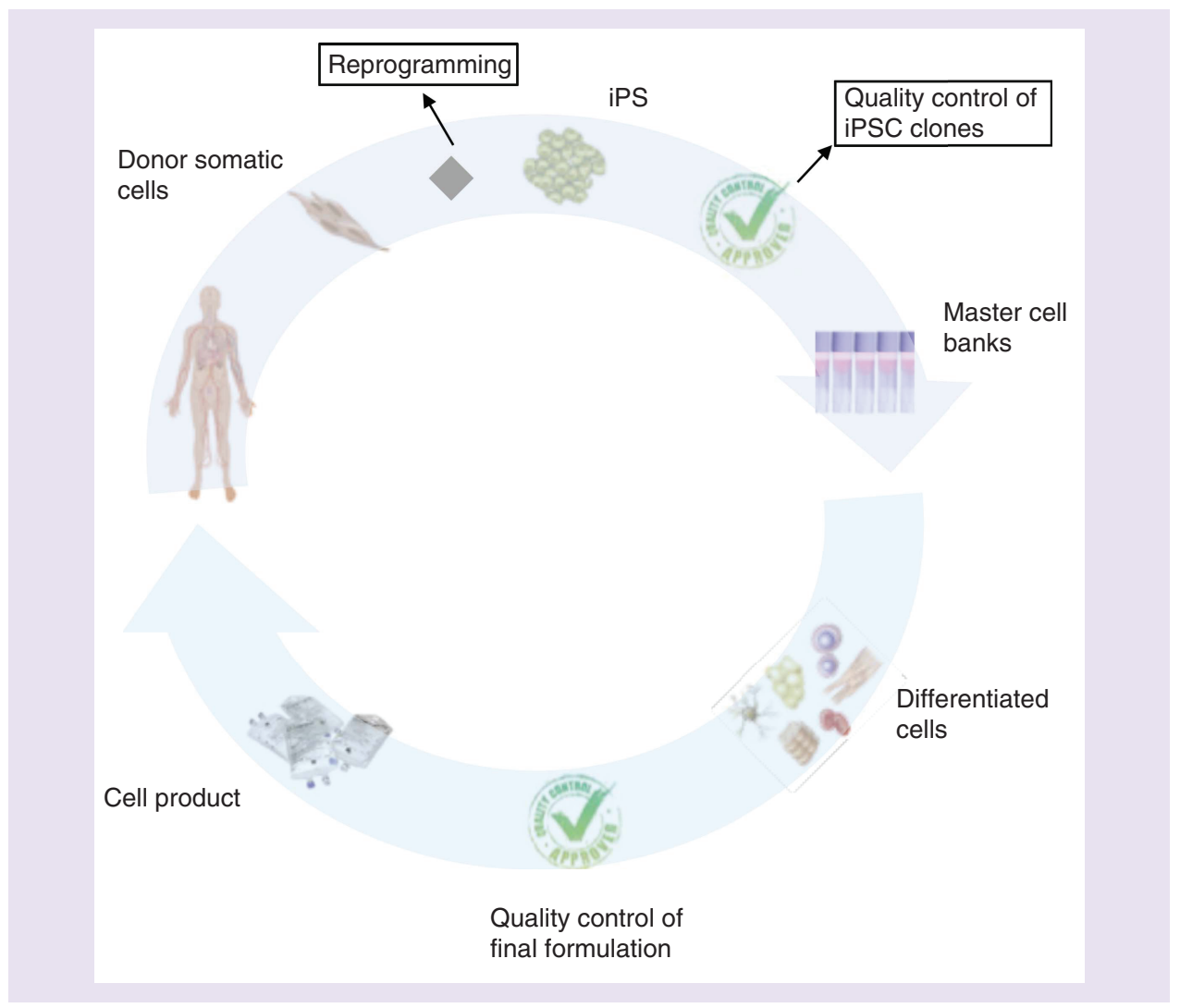


First draft submitted: 15 November 2018; Accepted for publication: 12 April 2019; Published online: 22 May 2019

Keywords: derivation • induced pluripotent stem cell • iPSC • KaryoStat ${ }^{\mathrm{TM}}$ assay $\bullet$ molecular characterization • Oncomine $^{T M}$ comprehensive assay v3 • PluriTest • reprogramming

Since the discovery of somatic reprogramming [1,2], induced pluripotent stem cells (iPSCs) have progressed rapidly toward applications in disease modeling, drug discovery and regenerative medicine [3-5]. The process of reprogramming involves delivery of key factors using integrating retro/lenti virus [1], ectopic expression using nonintegrating DNA [6] and non-DNA methods [7-10]. The use of iPSCs generated using integrating virus have limited application in translational research since the random genomic integration poses the risk of malignant transformation [11] via reactivation of integrated virus [12]. There is therefore greater focus on nonintegrating methods for the generation of footprint-free iPSCs such as mini circles [13], episomal plasmids [2], engineered viruses such as adenovirus [14], Sendai virus, a negative strand RNA virus [7], or synthetic molecules such as purified mRNA [8], miRNAs [9] and proteins [10]. While each method has its distinctive feature, the primary requirement for generating iPSCs is the need for robustness and reproducibility, best observed with Sendai virus (SeV)-based methods [15,16]. This method has shown success under stringent Good Manufacturing Practice (GMP) manufacturing conditions for the generation of patient-specific iPSCs [17]. Resulting iPSCs that are generated from diverse genetic backgrounds using different workflows and somatic cell types can lead to varied gene expression profiles, epigenetic landscapes, and karyotypes that can impact self-renewal properties, differentiation potential, and genomic integrity [18-23]. It is therefore critical that iPSC cell banks are generated using good cell culture practice and thoroughly characterized using standardized methods to ensure quality and safety [24]. An integrated workflow approach with high-quality reagents and robust methods is necessary for progressing iPSCs from research use to clinical and translational applications [25-30]. This study reports the development of workflow solutions that are specifically designed for the generation of iPSCs intended for use in clinical and translational research.

First, Sendai virus-based CTS CytoTune-iPS 2.1 Reprogramming Kit, manufactured in accordance with GMP requirements and free of animal origin components, was used to generate high-quality iPSCs under xeno-free culture conditions. Components used in translational workflows should avoid nonhuman animal origin components, such as fetal bovine serum or bovine serum albumin (BSA) because they are potential sources of adventitious agents. The reagents used for the generation of iPSCs should be manufactured under stringent quality systems to generate consistent products that ensure reliable outcomes. In addition, factors such as $c-M y c$, which is reported to have oncogenic potential [31], should be replaced with other members of the $M y c$ family such as $L-M y c$ or $c-M y c$ mutants (W136E and dN2) that are more specific and efficient in generating iPSCs but have little transformation activity [32]. The next generation reprogramming workflow described here considers these critical requirements and excludes any component of nonhuman origin (xeno-free), faithfully produces iPSC clones free of viral footprint based on a sensitive quantitative TaqMan qPCR-based assay of Sendai viral backbone and reduces the likelihood of oncogenic transformation by replacing $c-M y c$ with $L-M y c$.

Second, comprehensive molecular analysis platforms were utilized as fast and scalable alternatives to traditional methods for characterization of resulting iPSCs. Traditional methods of monitoring and confirming pluripotency rely on in vitro, cell-based assays [33-35], and in vivo, teratoma formation [36-39]. When PSC-derived cells are injected, they should be free of any residual pluripotent cells. The teratoma assay can be useful for verifying the absence if these undifferentiated cells. The semiquantitative nature of these assays renders them hard to standardize, thus impeding accuracy of stem cell characterization workflows, which has led to emergence of molecular assays [27,40]. Here, a panel of molecular assays used to establish pluripotency, genomic stability and safety were: PluriTest, a highly validated computational model of pluripotency that analyzes microarray-based global transcriptome data [41], and TaqMan hPSC Scorecard, a focused panel of select genes for quantitative assessment of pluripotency and trilineage differentiation using real-time qPCR [19,34,42]; determination of gross chromosomal copy number gain/loss or genetic mosaicism using whole genome array-based KaryoStat Assay or KaryoStat HD Assay; identification of variants associated with cancer-causing hotspot mutations including $T p 53$ using next generation sequencing-based oncomine comprehensive assay v3 (OCAv3); and authentication of the parental and resulting reprogrammed iPSC lines using microsatellite short tandem repeat (STR) analysis using AmpFLSTR Identifiler Direct PCR Amplification Kit. 
Finally, the different components described above were integrated for a seamless, xeno-free reprogramming workflow starting from isolation of dermal fibroblasts and blood-derived CD $34^{+}$and $\mathrm{CD} 3^{+}$pan T cells. Resulting footprint-free iPSCs were assessed to be pluripotent with normal karyotype, authenticated to be derived from the parental somatic cells and confirmed to lack any of the commonly known cancer-causing mutations. These results were found to be consistent across different sources of starting cell type. The ability to reliably create high-quality iPSCs in clinically relevant, xeno-free workflows is critical for enabling the smooth transition of iPSCs towards downstream clinical applications.

\section{Materials \& methods}

All materials were from Thermo Fisher Scientific unless stated otherwise.

All human cells were purchased from commercial companies, which obtained informed consent from donors and screened the material for adventitious agents.

\section{Culture of fibroblasts}

Commercially sourced adult human dermal fibroblasts (HDFa) were obtained from Gibco (Thermo Fisher Scientific catalog \# C1035C). Fibroblasts were cultured in one of the indicated fibroblast media and passaged every 3-5 days using TrypLE (catalog \# 12605010). Control fibroblast medium consisted of DMEM (catalog \# 10569010), 10\% fetal bovine serum (catalog \#16141079) and $0.1 \mathrm{mM}$ nonessential amino acids (NEAA; catalog \# 11140050). Xeno-free fibroblast medium 2 consisted of DMEM, 10\% human serum and $0.1 \mathrm{mM}$ NEAA. Xeno-free fibroblast medium 3 consisted of DMEM, 10\% Knockout Serum Replacement XenoFree (KSR-XF; catalog \# 12618012), $0.1 \mathrm{mM}$ NEAA, $12 \mathrm{ng} / \mathrm{ml}$ basic FGF (bFGF; catalog \# PHG0261), $1 \mu \mathrm{g} / \mathrm{ml}$ EGF (catalog \# PHG6045) and $1 \mu \mathrm{M}$ hydrocortisone (Sigma catalog \# H0888-1G). Xeno-free fibroblast medium 4 consisted of Essential 6 Medium (catalog \# A1516401), $12 \mathrm{ng} / \mathrm{ml} \mathrm{bFGF,} 1 \mu \mathrm{g} / \mathrm{ml}$ EGF and $1 \mu \mathrm{M}$ hydrocortisone. Xeno-free fibroblast medium 5 consisted of DMEM-F/12 (catalog \# 10565018), $0.48 \mathrm{mg} / \mathrm{ml}$ of additional sodium bicarbonate (catalog \# 25080094), 10\% KSR-XF, $0.1 \mathrm{mM}$ NEAA, $12 \mathrm{ng} / \mathrm{ml} \mathrm{bFGF}, 1 \mu \mathrm{g} / \mathrm{ml}$ EGF and $1 \mu \mathrm{M}$ hydrocortisone. Xeno-free fibroblast medium 6 consisted of Essential 8 Medium (catalog \# A1517001) and $1 \mu$ M hydrocortisone.

Growth rate was determined by counting the cells at each passage and plotting the number of days in culture against number of population doublings, which was calculated as $\log _{2}$ (\# cells harvested/\# cells plated). Transduction efficiency was determined using the CytoTune emGFP (emerald green fluorescent protein) Sendai reporter (catalog \# A16519). Fibroblasts were cultured for a minimum of three passages in the indicated medium and then transduced with the GFP Sendai virus at an MOI of 5. A total of $48 \mathrm{~h}$ after transduction, cells were analyzed by flow cytometry to determine the percentage of GFP-positive cells.

\section{Fibroblasts isolated in xeno-free conditions}

Sample of human dermis was obtained from Cardinal Biologicals (Tyler, TX, USA). A skin punch biopsy tool was used to isolate several small biopsies. These were further dissected with scalpels into smaller pieces $(\sim 1 \mathrm{~mm})$, which were placed into six-well plates coated with collagen matrix (catalog \# R011K; coating performed according to manufacturer instructions). Plates were placed in a $37^{\circ} \mathrm{C}$ incubator to allow the pieces to dry down and adhere to the plate. The appropriate fibroblast medium was gently added to the wells, and cells were cultured for 10-14 days, changing medium every 2-3 days until ready to passage. Cells were passaged using TrypLE and plated onto fresh collagen-coated plates.

\section{Culture of blood-derived cells \\ Peripheral blood mononuclear cells}

PBMCs were obtained from HemaCare (Van Nuys, CA, USA). PBMCs were cultured in StemPro34 serum-free medium (catalog \# 10639011) containing $200 \mathrm{mM}$ GlutaMax (catalog \# 35050061), $100 \mathrm{ng} / \mathrm{ml} \mathrm{SCF} \mathrm{(catalog} \mathrm{\#}$ PHC2116), $100 \mathrm{ng} / \mathrm{ml}$ FLT-3L (catalog \# PHC9415), $20 \mathrm{ng} / \mathrm{ml} \mathrm{IL-3} \mathrm{(catalog} \mathrm{\#} \mathrm{PHC0034)} \mathrm{and} 20 \mathrm{ng} / \mathrm{ml} \mathrm{IL-6}$ (catalog \# PHC0065).

\section{T cells}

$\mathrm{CD}^{+}$pan $\mathrm{T}$ cells were isolated from PBMCs using the Dynabeads FlowComp Human CD3 Kit (catalog \# 11365D). After isolation, cells were cultured in OpTmizer T cell expansion serum-free medium (catalog \# A1048501) containing 200 mM GlutaMax and 100 U/ml IL-2 (catalog \# PHC0021). 
CD34+ cells

Umbilical cord blood derived CD34+ ${ }^{+}$cells from pooled donors were obtained from Gibco (catalog \# A14059). CD34 ${ }^{+}$cells were cultured in OpTmizer SFM containing $200 \mathrm{mM}$ GlutaMax, $100 \mathrm{ng} / \mathrm{ml} \mathrm{SCF,} 50 \mathrm{ng} / \mathrm{ml} \mathrm{IL-3} \mathrm{and}$ $25 \mathrm{ng} / \mathrm{ml}$ GM-CSF (catalog \# PHC2015).

\section{iPSC generation}

Somatic cells were reprogrammed using Sendai virus based CytoTune iPS 2.0 kit (CytoTune 2.0; catalog \# A16517) and CTS CytoTune iPS 2.1 kit (CytoTune 2.1; catalog \# A34546). An alternate CytoTune2.0L with KOS, L-Myc and Klf4 was used in an initial evaluation.

\section{Fibroblasts}

HDFs were plated in a six-well plate 2-3 days prior to transduction to achieve a density of 2e5-3e 5 cells per well. Cells were transduced with the indicated set of Sendai vectors at an MOI of 5-5-3 (KOS-Myc-Klf4). Virus was removed after $24 \mathrm{~h}$, and cells were cultured in fibroblast medium for a total of 7 days, fed every other day. On day 7 after transduction, cells were harvested with TrypLE and plated in the appropriate fibroblast medium onto vitronectin (catalog \# A14700) or Laminin-521 (LN521; catalog \# A29248) coated plates. The following day, medium was changed to Essential 8 Medium, and cells were fed daily until day 21. At day 21, cells were either analyzed for reprogramming efficiency using a terminal alkaline phosphatase (AP) stain (Vector Labs catalog \# SK5100), or for further culture individual colonies were manually dissected and transferred to fresh VTN-N-coated dishes.

\section{Peripheral blood mononuclear cell}

PBMCs were cultured for 4 days after thaw, and on the day of transduction, $3 \mathrm{e} 5$ cells were combined with the indicated set of Sendai vectors at a MOI of 5-5-3 (KOS-Myc-Klf4). Cells and virus were centrifuged at $1000 \times \mathrm{g}$ for $30 \mathrm{~min}$ and then incubated overnight. Virus was removed after $24 \mathrm{~h}$, and cells were cultured in suspension until day 3 after transduction. At day 3, cells were plated onto VTN-N or LN521-coated plates in PBMC medium containing no cytokines. Cells were fed every other day, and on day 7 half the medium was replaced with Essential 8 Medium. On Day 8, medium was fully changed to Essential 8, and cells were fed every day thereafter. Reprogramming was ended when colonies were fully grown and before the emergence of extensive differentiation, typically at day 16-18 after transduction. Cells were either analyzed for reprogramming efficiency using a terminal AP stain or for further culture individual colonies were manually dissected and transferred to fresh VTN-N-coated dishes.

CD34+ cells

Umbilical cord blood-derived CD34+ ${ }^{+}$cells were cultured for 3 days after thaw, and on the day of transduction, 1e 5 cells were combined with the indicated set of Sendai vectors at an MOI of 5-5-3 (KOS-Myc-Klf4), and $4 \mu \mathrm{g} / \mathrm{ml}$ of Polybrene (Millipore catalog \# TR-1003-G). Virus was removed after $24 \mathrm{~h}$, and cells were cultured in suspension until day 3 after transduction. At day 3, cells were plated onto VTN-N or LN521-coated plates in CD34+ medium containing no cytokines. Cells were fed every other day, and on day 7 half the medium was replaced with Essential 8 Medium. On Day 8, medium was fully changed to Essential 8, and cells were fed every day thereafter. Reprogramming was ended when colonies were fully grown and before the emergence of extensive differentiation, typically at day 16-18 after transduction. Cells were either analyzed for reprogramming efficiency using a terminal AP stain, or for further culture individual colonies were manually dissected and transferred to fresh VTN-N-coated dishes.

\section{T cells}

$\mathrm{CD}^{+}$pan T cells were activated using Dynabeads Human T-Expander CD3/CD28 beads (catalog \# 11141D). Beads were added to cells at a ratio of 3:1, and cells were cultured for 5-7 days, adding fresh T cell medium every other day. On the day of transduction, $3 \mathrm{e} 5$ cells were combined with the indicated set of Sendai vectors at an MOI of 5-5-3 (KOS-Myc-Klf4). Virus was removed after $24 \mathrm{~h}$, and cells were cultured in suspension until day 2 after transduction. At day 2, CD3/CD28 beads were removed, and cells were plated onto VTN-N or LN521 coated plates in T-cell medium. On day 3, medium was changed to Essential 8, and cells were fed every day thereafter. Reprogramming was ended when colonies were fully grown and before the emergence of extensive differentiation, typically at day 14-18 after transduction. Cells were either analyzed for reprogramming efficiency using a terminal 
AP stain, or for further culture individual colonies were manually dissected and transferred to fresh VTN-N-coated dishes.

\section{Matrix coating}

Vitronectin coating was performed according to manufacturer instructions; the stock solution was diluted 1:1000 in Dulbecco's phosphate-buffered saline without calcium and magnesium (catalog \# 14190144) to a final concentration of $0.5 \mu \mathrm{g} / \mathrm{ml}$, adding the solution to tissue-culture treated plates and incubating at $37^{\circ} \mathrm{C}$ for $60 \mathrm{~min}$. Laminin- 521 (LN-521) coating was performed according to manufacturer instructions; the stock solution was diluted 1:40 in Dulbecco's phosphate-buffered saline with calcium and magnesium (catalog \# A14040133) to a final concentration of $2.5 \mu \mathrm{g} / \mathrm{ml}$, adding the solution to tissue-culture treated plates, and incubating at $37^{\circ} \mathrm{C}$ for $2 \mathrm{~h}$.

\section{iPSC culture}

Early passage (passage 1-passage 5) iPSC were passaged using manual dissection of colonies. Once iPSC clones were established, they were routinely cultured on VTN-N coated dishes in Essential 8 medium, and cells were split every 3-5 days using $0.5 \mathrm{mM}$ EDTA (catalog \# AM9912). iPSC clones were carried to at least passage 10 before banking and characterization. For analysis of differentiation potential, EBs were formed as follows; PSC were harvested using $2 \mathrm{mg} / \mathrm{ml}$ Collagenase IV (catalog \# 17104019) and cultured in suspension using nontissue culture treated dishes, in PSC medium consisting of DMEM/F-12, 20\% Knockout serum replacement (catalog \# 10828028), 0.1 mM NEAA, 55 MM 2-mercaptoethanol (catalog \# 21985023) and $10 \mathrm{ng} / \mathrm{ml} \mathrm{bFGF}$. After $24 \mathrm{~h}$, cells were cultured in PSC medium without bFGF and cultured further for 6 more days and then harvested for ScoreCard analysis, or for 2 more days, then plated onto Geltrex (catalog \# A1413201) coated 24-well plates and cultured for 18 more days before immunostaining.

\section{Immunostaining}

For in situ staining, cells were fixed with 4\% paraformaldehyde for 15-20 min at room temperature, blocked and permeabilized using 5\% normal goat serum (catalog \# PCN5000) and 0.1\% Triton X-100 (catalog \# HFH10) for $45 \mathrm{~min}$ at room temperature, then stained with primary antibodies for $1 \mathrm{~h}$ at room temperature or overnight at $4^{\circ} \mathrm{C}$, washed with blocking/permeabilizing solution and stained with secondary antibodies for $60 \mathrm{~min}$ at room temperature. Primary antibodies used: undifferentiated PSC marker staining - TRA-1-60 (catalog \# 411000), SSEA4 (catalog \# 414000), SOX2 (catalog \# 481400), OCT4 (catalog \# A13998); embryoid body staining $-\alpha$ fetoprotein (AFP; catalog \# 1830003), smooth muscle actin (SMA; catalog \# 180106), and $\beta$-III tubulin (BIIITub; catalog \# 480011). Secondary antibodies used were goat anti-mouse IgG $(\mathrm{H}+\mathrm{L})$, highly cross-adsorbed secondary antibody, Alexa Fluor 488 (Catalog \# A11029), or Alexa Fluor 594 (Catalog \# A11032).

For staining followed by flow cytometry analysis, PSC were harvested using TrypLE and then stained with conjugated antibodies Tra-1-60 AlexaFluor488 (BD catalog \# 560173), and SSEA1 AlexaFluor647 (BD catalog \# 560120) for $45 \mathrm{~min}$ at room temperature. Cells were washed twice and analyzed on the Attune NxT Flow Cytometer.

\section{TaqMan hPSC scorecard assay (scorecard)}

RNA was extracted using TRIzol (catalog \# 15596018) and digested with DNase I (catalog \# 18068015) before being used for cDNA synthesis. Template cDNA was prepared from $1 \mu \mathrm{g}$ of RNA using the High-Capacity cDNA Reverse Transcription kit (catalog \# 4368814) according to the manufacturer's protocol. cDNA was diluted further with nuclease-free water and TaqMan Gene Expression Master Mix (catalog \# 4369016). ScoreCard (catalog \# A15876) assays were run according to manufacturer's instructions on a QuantStudio 12 K Flex Real-Time PCR System and data was analyzed with ScoreCard Thermo Fisher cloud-based software.

\section{G-Band karyotype}

G-Band karyotype of iPSC clones and primary cells was performed by Cell Line Genetics (MI, USA)

\section{KaryoStat assay}

Genomic DNA (gDNA) was extracted using PureLink Genomic DNA Mini Kit (catalog \# K182000) and estimated by Qubit dsDNA BR Assay Kits. gDNA was processed according to manufacturer's instructions. Briefly, 250 ng gDNA was digested with Nsp I restriction enzyme. Digested DNA was adapter ligated and PCR amplified. 
DNA was then fragmented and labeled with biotin. Fragmented-labeled DNA was hybridized onto GeneChip arrays in a GeneChip Hybridization Oven 645 overnight. Chips were washed and stained simultaneously using GeneChip Fluidics Station 450 and scanned using GeneChip Scanner 30007 G. Data were analyzed using ChAS 3.2. The raw data were processed using Genotyping Console v 4.0 and Chromosome Analysis Suite 3.2 with NetAffx na33.1 (UCSC GRCh37/hg19), and the output data were interpreted with the UCSC Genome Browser (https://genome.ucsc.edu/; GRCh37/hg19 assembly). The functions of the genes, which were located within the region of the genomic imbalance, were retrieved from the GeneCards (www.genecards.org) and OMIM (www.ncbi.nlm.nih.gov/omim) databases.

\section{PluriTest assay}

RNA was extracted using TRIzol and digested with DNase I. Purified RNA was estimated by Qubit RNA BR Assay Kit. RNA was processed for PrimeView Human Gene Expression Assay according to manufacturer's instruction. Briefly, 100 ng RNA was amplified via in vitro transcription primed through $3^{\prime}$ poly dT oligo primer. Amplified RNA was fragmented and labeled with biotin. Fragmented-labeled RNA was then hybridized overnight onto PrimeView Human Gene Expression microarrays in a GeneChip Hybridization Oven 645. Chips were washed and stained simultaneously using GeneChip Fluidics Station 450 and scanned using Affymetrix GeneChip Scanner 30007 G. After a preliminary QC check of CEL files by Transcriptome Analysis Console 4.0, data analysis was performed at https://pluritest.org, a cloud-based software analysis tool.

\section{SeV detection assay}

RNA was extracted using TRIzol and digested with DNase I. cDNA synthesis was performed with 1 ug of RNA in a $20 \mu \mathrm{l}$ reaction using high-capacity cDNA reverse transcription kit according to the manufacturer's protocol. The cDNA was then diluted with nuclease-free water. PCR amplification was performed using TaqMan Gene Expression Master Mix in QuantStudio 12 K Flex Real Time PCR System. Approximately 20 ng cDNA was used per well. A 5-log full panel of serially diluted $\mathrm{SeV}$ plasmid standards was prepared using diluent TE ( $\mathrm{pH}$ 8.0) containing $10 \mathrm{ng} / \mu \mathrm{l}$ Lambda DNA (catalog \# SD0011). SeV copy number was extracted from the standard curve. ActB served as a normalizing control.

\section{Oncomine comprehensive assay v3}

Genomic DNA was extracted using PureLink Genomic DNA Mini Kit and estimated by Qubit dsDNA highsensitivity (HS) assay kits. Libraries were prepared according to instructions provided with OCAv3. Briefly, approximately $20 \mathrm{ng}$ DNA was PCR amplified and partially digested. Barcoded adapters are then ligated to the digested amplicons. Individual libraries were quantified and pooled together at equal molar ratio. Ion Chef System was primarily used for sample preparation and loading. Samples were loaded onto Ion 540 Chips and ran on Ion GeneStudio S5 system. AcroMetrix Oncology Hotspot Control included in this assay served as positive control, a highly multiplexed, proprietary DNA quality control designed to enable labs to control the hundreds of amplicons targeted by next-generation sequencing (NGS) panels. It contains over 500 mutations from the Catalogue of Somatic Mutations in Cancer (COSMIC) database and has five variant types of varying nucleotide lengths. The 53 genes represented in the AcroMetrix Oncology Hotspot Control are: ABL1, AKT1, ALK, APC, ATM, BRAF, CDH1, CDKN2A, CSF1R, CTNNB1, EGFR, ERBB2, ERBB4, EZH2, FBXW7, FGFR1, FGFR2, FGFR3, FLT3, FOXL2, GNA11, GNAQ, GNAS, HNF1A, HRAS, IDH1, IDH2, JAK2, JAK3, KDR, KIT, KRAS, MAP2K1, MET, MLH1, MPL, MSH6, NOTCH1, NPM1, NRAS, PDGFRA, PIK3CA, PTEN, PTPN11, RB1, RET, SMAD4, SMARCB1, SMO, SRC, STK11, TP53, VHL.

\section{Cell line authentication using short tandem repeats}

$1 \mu \mathrm{l}$ of extract was PCR amplified using the Identifiler Direct STR profiling kit (catalog \# 4467831), according to manufacturer's instructions. $1 \mu \mathrm{l}$ of PCR product was combined with HiDi formamide and LIZ600 sizing markers, denatured at $95^{\circ} \mathrm{C}$ for $3 \mathrm{~min}$, and analyzed on a SeqStudio Genetic Analyzer using conditions in the default 'fragment analysis' module supplied on the instrument. One capillary was run with the allelic ladder provided in the Identifiler Direct kit. Alleles present in each sample were determined using GeneMapper 5 software and the appropriate panels and bins. 


\section{Results}

\section{Development of optimal xeno-free reprogramming workflows}

A key requirement for translational research is the use of high-quality raw materials devoid of adventitious agents which can be easily integrated into xeno-free workflows. In accordance with this, a Sendai-virus based reprogramming kit was developed that is free of components such as BSA or FBS, manufactured in accordance with GMP requirements and modified to replace $c-M y c$ with the less oncogenic $L-M y c[32,43]$. This was integrated into an optimized xeno-free workflow for reprogramming fibroblasts and blood cells (Figure 1A). In addition to the change to $L-M y c$, further efforts to increase the safety of the reprogramming system were made by eliminating any xeno-based materials. For the reprogramming kit itself, BSA was removed from the viral dilution buffer. Results from this study indicated that reprogramming of HDFa with $L-M y c$ (CytoTune 2.0L) resulted in an approximately threefold decrease in reprogramming efficiency. When the xeno-free Sendai kit (CytoTune 2.1) was compared with the kit containing L-Myc (CytoTune 2.0L), no significant difference was found in the resulting reprogramming efficiency (Figure 1B). This suggests that the xeno-free formulation did not affect reprogramming efficiency, and any decreased efficiency observed with CytoTune 2.1 was primarily due to the substitution of $L-M y c$ for $c-M y c$. Similarly, reprogramming of blood-derived cells with CytoTune 2.1 resulted in a 1- to 2.5-fold decrease in reprogramming efficiency, compared with CytoTune 2.0 reprogramming (Figure 1C). Despite the reduction in reprogramming efficiency, consistency in generation of iPSCs was observed across the three different donor cell types tested.

\section{Identification of xeno-free fibroblast medium for isolation, expansion \& reprogramming of HDFs}

Typical fibroblast culture medium includes supplementation with FBS, and fibroblast reprogramming protocols require culture of the cells in a suitable fibroblast medium. Since the goal was to develop entirely xeno-free reprogramming workflows, it was necessary to develop a xeno-free fibroblast medium that would support the robust fibroblast growth that is needed for successful reprogramming. The most obvious substitute for FBS would be human serum, but when HDFa cultured in a medium containing human serum were transduced with a GFPexpressing Sendai virus, the transduction efficiency was severely impacted (Supplementary Figure 1A). Based on xeno-free fibroblast media formulations reported earlier [26,44-46], five different formulations were evaluated for Sendai-GFP transduction efficiency and fibroblast growth rate (Supplementary Figure 1B). The top two xenofree media along with FBS-containing medium as control were further evaluated for growth rate, Sendai-GFP transduction efficiency, and reprogramming efficiency with CytoTune 2.0. Transduction efficiency for both media was comparable with that of the control FBS-containing medium (Figure 2A). However, growth rate for xeno-free medium 6 was higher than xeno-free media 5 (Figure 2B) and the reprogramming efficiency for both xeno-free media was relatively lower compared with FBS containing control (Figure 2C), albeit to a lower extent with xeno-free media 5. Taken together, these results led to the use of xeno-free medium 5 as the fibroblast medium of choice in all further experiments. In order to establish a completely xeno-free fibroblast reprogramming protocol, HDFs were isolated and expanded in xeno free media 5. When cultured on human collagen matrix, the cells displayed typical fibroblast morphology (Figure 2D) and no karyotypic abnormalities were detected by G-banded karyotyping (Figure 2E). These fibroblasts (HDFa XF5) were then reprogrammed with the CTS CytoTune 2.1 kit, and five iPSC (CFa923 clones $\mathrm{C} 1-\mathrm{C} 5)$ were isolated, expanded and characterized.

\section{Confirmation of SeV elimination \& pluripotency in iPSCs generated using xeno-free workflows}

Although Sendai is a nonintegrating negative strand RNA virus, it is critical to examine if there is any virus left in iPSC clones. Presence of any residual viral backbone or transcript would make an iPSC line unsuitable for clinical usage. An in-house developed custom Sendai quantitation kit was employed that utilizes validated TaqMan primers with a high level of specificity and sensitivity. This assay can detect and quantify as low as one copy with $95 \% \mathrm{CI}$ $(\mathrm{LoQ}=1)$. The assay detected very high $\mathrm{SeV}$ copy number in cells 4-7 days after transduction; however, analysis of the iPSC clones at P10 failed to detect the presence of any trace of $\mathrm{SeV}$ backbone or transcript. This result confirms that the iPS clones are $\mathrm{SeV}$ footprint-free (Table $1 \mathrm{~A} \& \mathrm{~B}$ ).

iPSC clones generated using CTS CytoTune 2.1 from fibroblast derived from donor cells in completely xeno-free workflow were assessed to confirm pluripotency based on marker expression and differentiation potential. Selected CFa923 clones C2-C4 stained for known pluripotent makers TRA-1-60, SSEA4, OCT4 and SOX2 demonstrated uniform pattern of staining consistent with pluripotent stem cells (Figure 3A). Clones were assessed for growth rate (Supplementary Figure 2A) and were found to have similar rates among each other and similar rates compared with a separate control iPSC line. Flow cytometry analysis was used to quantitate marker expression for Tra-1-60 and 
(A)

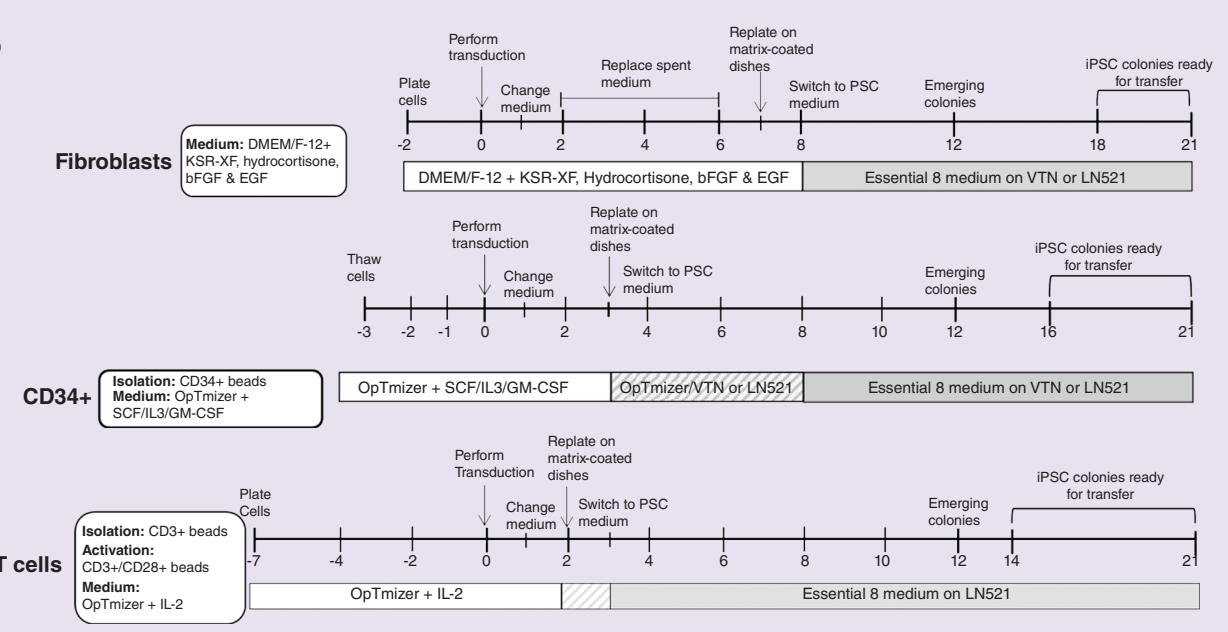

(B)

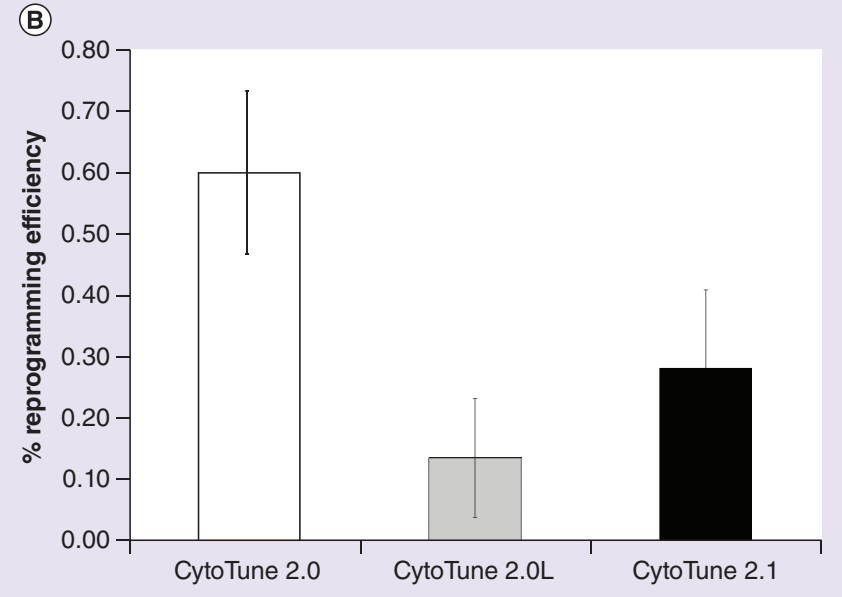

(C)

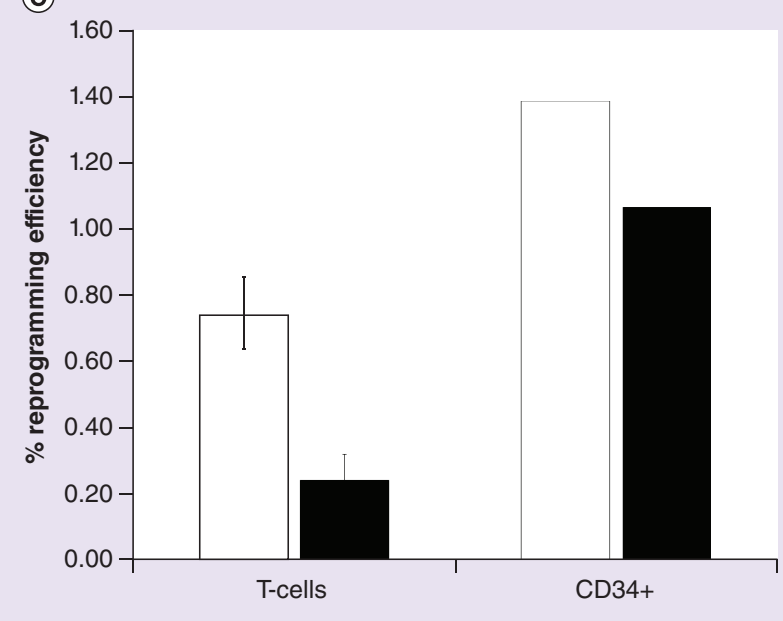

Figure 1. Comparison of reprogramming kits. (A) Schematics outlining the reprogramming protocols for each of the different cell types. (B) The HDFa reprogramming efficiency was compared between three different CytoTune kit types. At day 21 after transduction, cells were analyzed for reprogramming efficiency using a terminal alkaline phosphatase (AP) stain, and reprogramming efficiency was calculated as the percent of AP positive colonies relative to the number of cells plated at day 7. Three different adult human dermal fibroblast donor lots were reprogrammed with each kit type. Error bars indicate standard deviation (s.d.; $n=3$ ). (C) Reprogramming efficiency was compared between CytoTune 2.0 and CytoTune 2.1 across two different blood-derived cell types; $\mathrm{CD}^{+}$Pan T Cells (two different donors reprogrammed) and $\mathrm{CD}_{4} 4^{+}$cells (one pooled donor sample reprogrammed). At day 14-18 after transduction, cells were analyzed for reprogramming efficiency using a terminal AP stain, and reprogramming efficiency was calculated as the percent of AP positive colonies relative to the number of cells plated at day 2 or day 3 (for T cells and $\mathrm{CD}_{3} 4^{+}$cells, respectively). For T-cells, error bars indicate $\mathrm{s} . \mathrm{d} .(\mathrm{n}=2)$.

iPSC: Induced pluripotent stem cell; PSC: Pluripotent stem cell. 
(A)

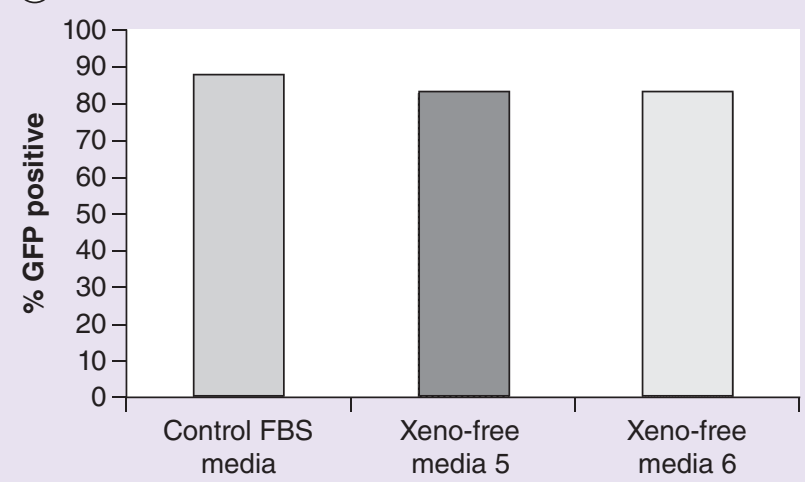

(B)

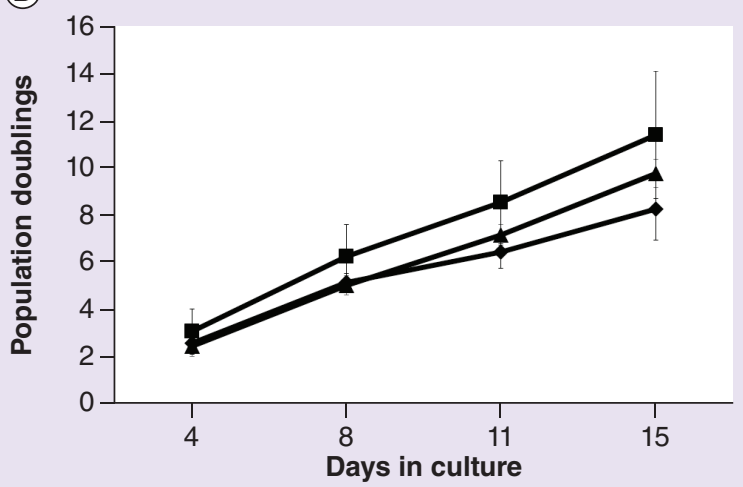

(c)

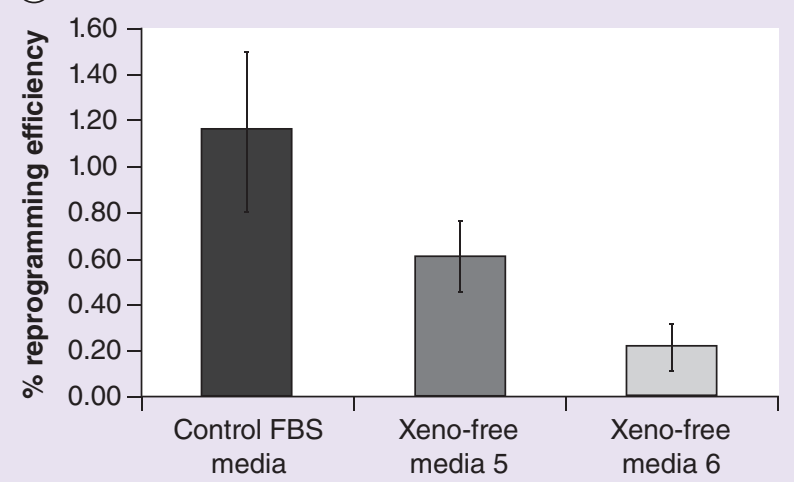

(D)

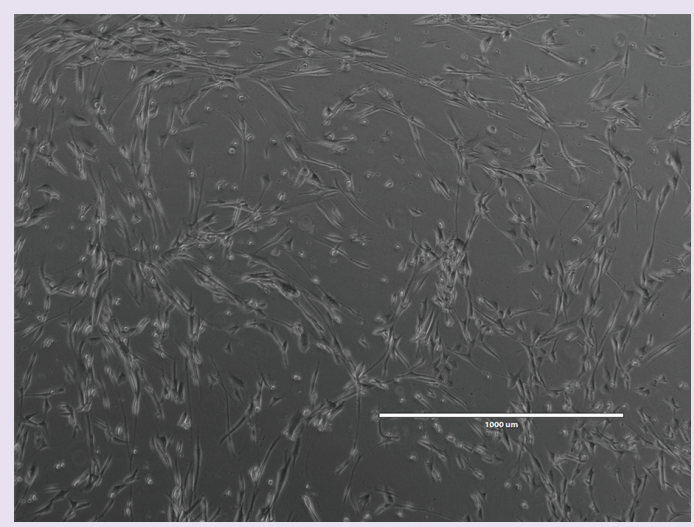

FBS medium $\bullet$ Xeno-free medium $5 \bullet$ Xeno-free medium 6

(E)

Result: 46,XX

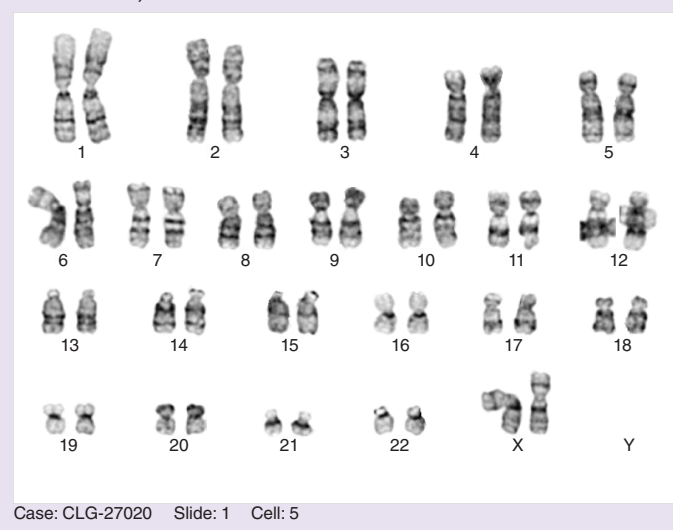

Figure 2. Development of a xeno-free fibroblast medium. Candidates for xeno-free fibroblast media were evaluated for (A) Sendai GFP transduction efficiency as measured by flow cytometry. (B) Growth rate was determined by counting the cells at each passage and plotting the number of days in culture against number of population doublings, which was calculated as $\log _{2}$ (\# cells harvested/\# cells plated). Error bars indicate standard deviation (s.d.; $n=3$ ). (C) Reprogramming efficiency with CytoTune 2.0. At day 21 after transduction, cells were analyzed for reprogramming efficiency using a terminal alkaline phosphatase (AP) stain, and reprogramming efficiency was calculated as the percent of AP positive colonies relative to the number of cells plated at day 7 . Error bars indicate s.d. $(n=3)$. Xeno-free Medium 5 was the best performing medium and was used to derive and expand human dermal fibroblasts from a skin biopsy. (D) Xeno-free-derived fibroblasts displayed typical morphology, and a (E) normal karyotype, as assessed by G-band. FBS: Fetal bovine serum; GFP: Green fluorescent protein. 
(A)
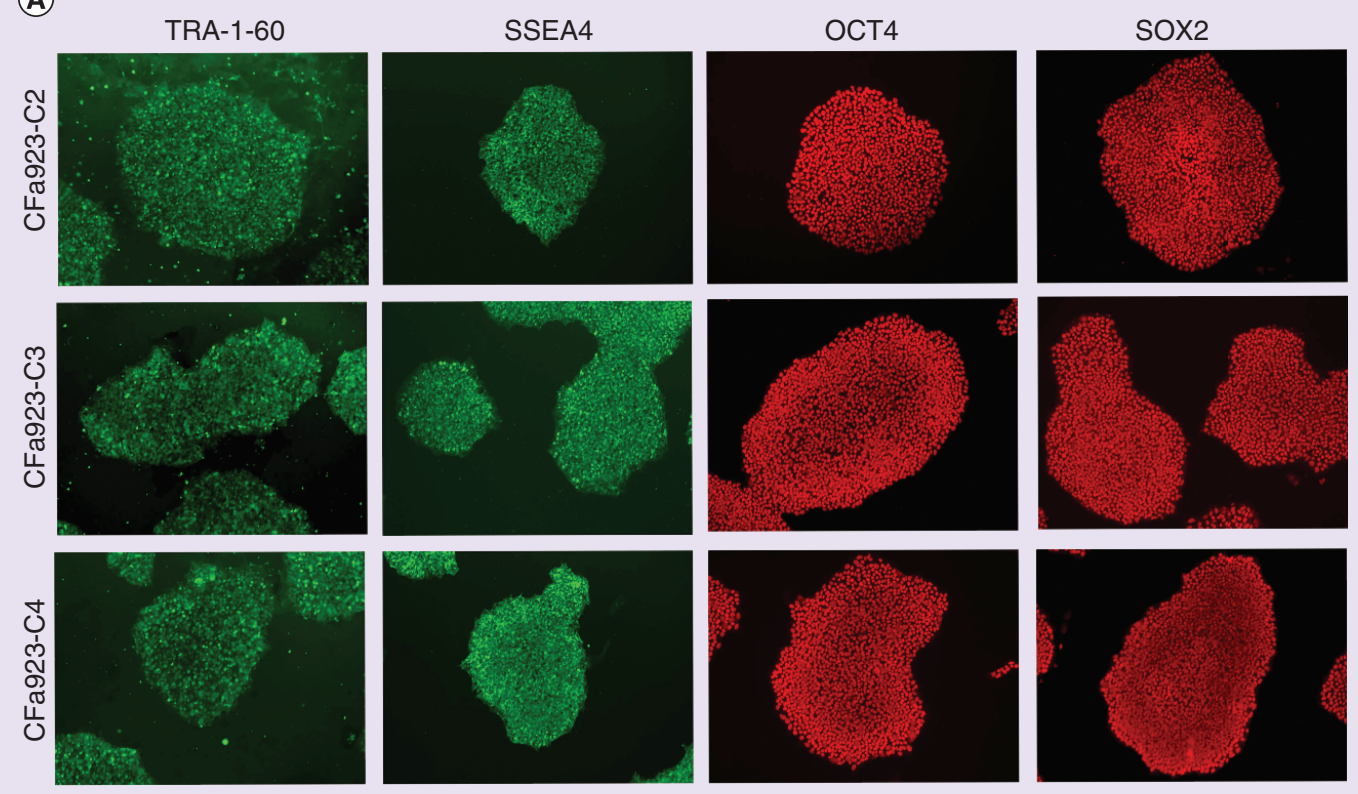

(B)

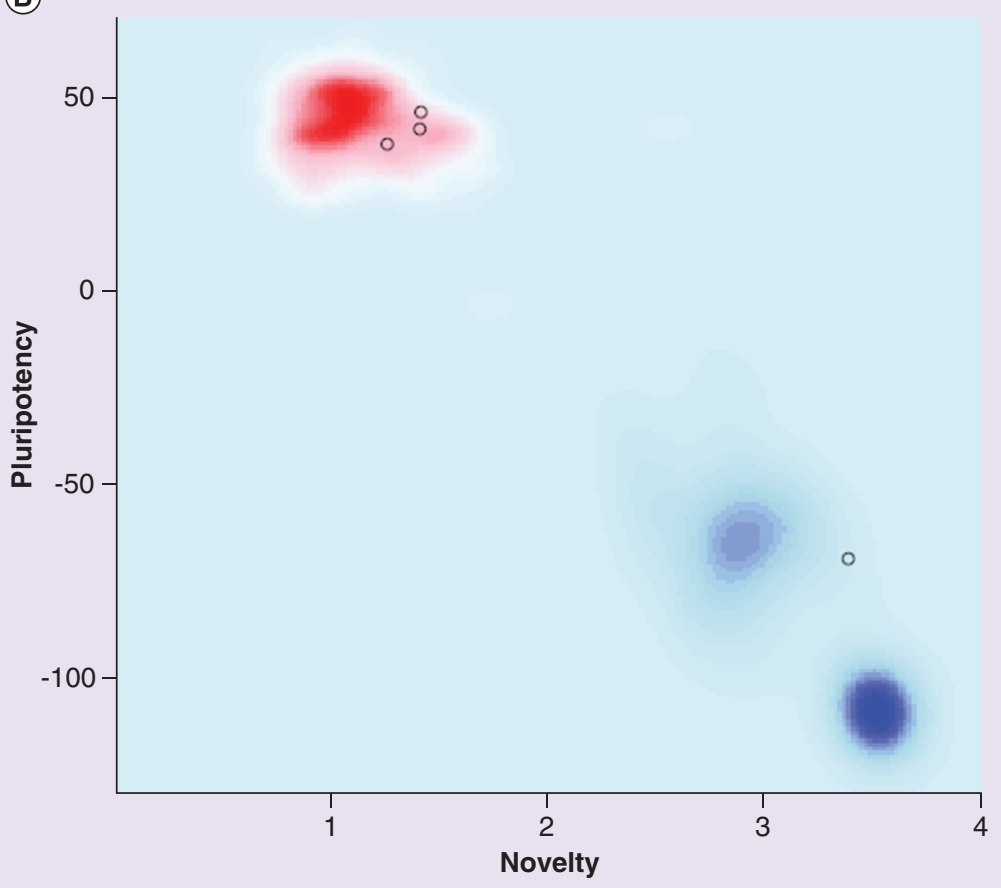

Figure 3. Further characterization of induced pluripotent stem cells clones generated from the xeno-free derived fibroblasts. (A) Three different induced pluripotent stem cell (iPSC) clones were stained for typical markers of pluripotency - Tra-1-60, SSEA4, OCT4 and SOX2 - and visualized under fluorescence microscopy. (B) iPSC clones and the parental fibroblasts were analyzed using the PluriTest assay. (C) To assess trilineage differentiation potential, iPSC clones were randomly differentiated as embryoid bodies for 21 days and then stained for markers representative of the three germ layers; AFP (endoderm), SMA (mesoderm) and $\beta$ IIITub (ectoderm). (D) Trilineage differentiation potential of the iPSC clones was also analyzed for using the ScoreCard qPCR assay, using both undifferentiated samples (PSC) and samples from 7 day embryoid body differentiation.

AFP: $\alpha$-fetoprotein; PSC: Pluripotent stem cell; SMA: Smooth muscle actin; Tub: Tubulin. 


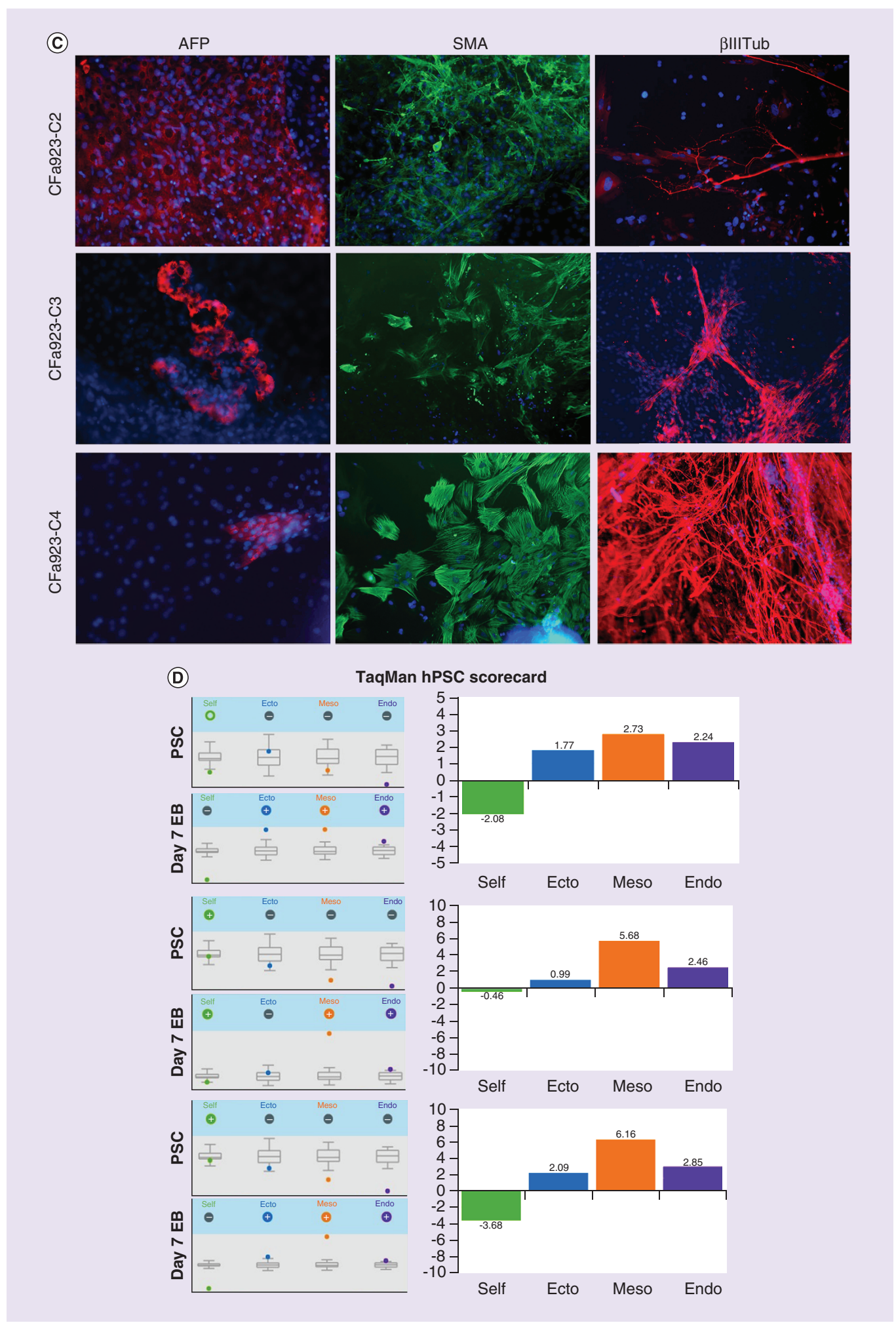

Figure 3. Further characterization of induced pluripotent stem cells clones generated from the xeno-free derived fibroblasts (cont.). (A) Three different induced pluripotent stem cell (iPSC) clones were stained for typical markers of pluripotency - Tra-1-60, SSEA4, OCT4 and SOX2 - and visualized under fluorescence microscopy. (B) iPSC clones and the parental fibroblasts were analyzed using the PluriTest assay. (C) To assess trilineage differentiation potential, iPSC clones were randomly differentiated as embryoid bodies for 21 days and then stained for markers representative of the three germ layers; AFP (endoderm), SMA (mesoderm) and $\beta$ IIITub (ectoderm). (D) Trilineage differentiation potential of the iPSC clones was also analyzed for using the ScoreCard qPCR assay, using both undifferentiated samples (PSC) and samples from 7 day embryoid body differentiation.

AFP: $\alpha$-fetoprotein; PSC: Pluripotent stem cell; SMA: Smooth muscle actin; Tub: Tubulin. 
Table 1A. TaqMan quantitative PCR-based assay for absolute quantitation of Sendai virus copy number.

\begin{tabular}{|lllll|}
\hline Sample name & Sendai Ct & Sendai copy number & ActB Ct & ActB copy number \\
\hline $923-$ XF5-1 & Undetermined & N/A & 25.0 & 58,000 \\
\hline $923-$ XF5-2 & Undetermined & N/A & 25.0 & 58,000 \\
\hline $923-X F 5-3$ & Undetermined & N/A & 25.1 & 53,000 \\
\hline $923-X F 5-4$ & Undetermined & N/A & 25.0 & 60,000 \\
\hline $923-X F 5-5$ & Undetermined & N/A & 24.8 & 66,000 \\
\hline Positive control & 20.8 & 77,000 & 25.7 & 34,000 \\
\hline
\end{tabular}

\begin{tabular}{|c|c|c|}
\hline Copy number (standard) & Sendai Ct & ActB Ct \\
\hline 1 & 37.3 & 40.6 \\
\hline 8 & 34.2 & 37.8 \\
\hline 40 & 32.0 & 35.6 \\
\hline 200 & 30.0 & 33.4 \\
\hline 1000 & 27.2 & 31.0 \\
\hline 5000 & 24.9 & 28.5 \\
\hline 25,000 & 22.3 & 26.0 \\
\hline
\end{tabular}

SSEA1 (a negative marker) (Supplementary Figure 2B), and the clones analyzed showed $>70 \%$ Tra- 160 expression and $<5 \%$ SSEA1 expression. Global transcriptome analysis of these clones was performed using PrimeView Human Gene Expression microarray followed by PluriTest, an online bioinformatic assay that compares the gene expression profile with an extensive reference set of $>450$ cell and tissue types [41]. The analysis tool confirms pluripotency via two separate scores: pluripotency and novelty. The pluripotency score is an indication of how strongly a model-based pluripotency signature is expressed in the samples analyzed. The novelty score indicates the general model fit for a given sample with reference to stem cells as well as other cell types. Generally, a pluripotency score above 20 and a novelty score below 1.7 indicate that cells resemble typical hPSCs and that they are similar to normal hPSCs, respectively [41]. Figure 3B illustrates that all three reprogrammed clones cluster together with a very high pluriscore $(>35)$ and low novelty score $(<1.4)$ (Supplementary Table 1) while the parental line displayed a low pluriscore and a high novelty score, typical of nonpluripotent cells.

Trilineage differentiation potential of the iPSCs was further assessed via spontaneous differentiation of embryoid bodies (EBs) followed by immunostaining with antibodies against lineage-specific markers, representative of the three germ layers. The three iPSC clones differentiated for 3 weeks showed expression of $\alpha$ fetoprotein, smooth muscle actin and $\beta$ tubulin representing endoderm, mesoderm and ectoderm, respectively (Figure 3C). These samples were further analyzed using TaqMan hPSC Scorecard assay, a focused qPCR gene expression panel of 93 genes coupled with a cloud-based data analysis tool that assess pluripotency and trilineage differentiation status of undifferentiated and differentiated samples in comparison to a reference set of 13 well-characterized embryonic stem cell (ESC) and iPSC lines [19]. As Figure 3D indicates, all clones showed presence of self-renewal and absence of lineage markers (endoderm, ectoderm, mesoderm) in undifferentiated cells (PSC) and vice versa with absence of self-renewal and presence of lineage markers in corresponding day 7 EB samples (day $7 \mathrm{~EB}$ ). This is further represented as a differentiation index plot that shows a decrease in self-renewal and upregulation of lineage markers in differentiating cells relative to undifferentiated cells for all clones confirming their potential to differentiate into cell types representing the three germ layers.

\section{Genomic integrity \& authentication of iPSC generated using xeno-free workflow}

To determine if long-term culturing and passaging impacted the genomic integrity of the iPSC clones, iPSC clones $\mathrm{CFa} 923-\mathrm{C} 3$ and $\mathrm{CFa} 923-\mathrm{C} 4$ were karyotyped using standard G-banding. Cytogenetic analysis was performed on 20 G-banded metaphase cells. For both the clones, 19 cells demonstrated apparently normal female karyotype and one cell demonstrated a nonclonal chromosome, which was determined to be an artifact of culture (Figure 4A). These clones and additional iPSC lines were further examined using KaryoStat and KaryoStat HD, whole-genome 
(A)

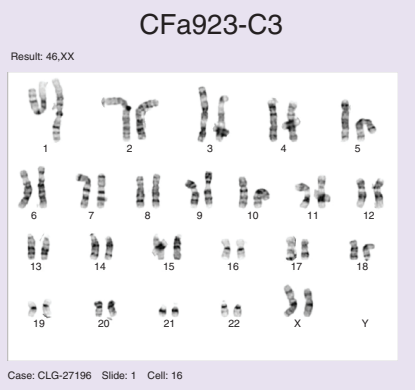

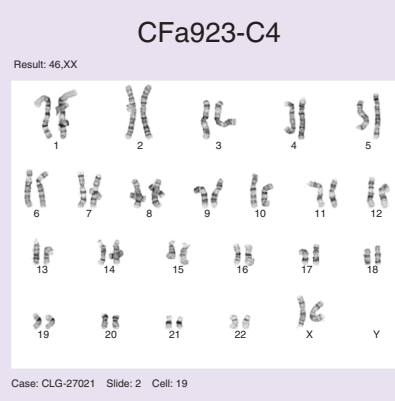

(B)
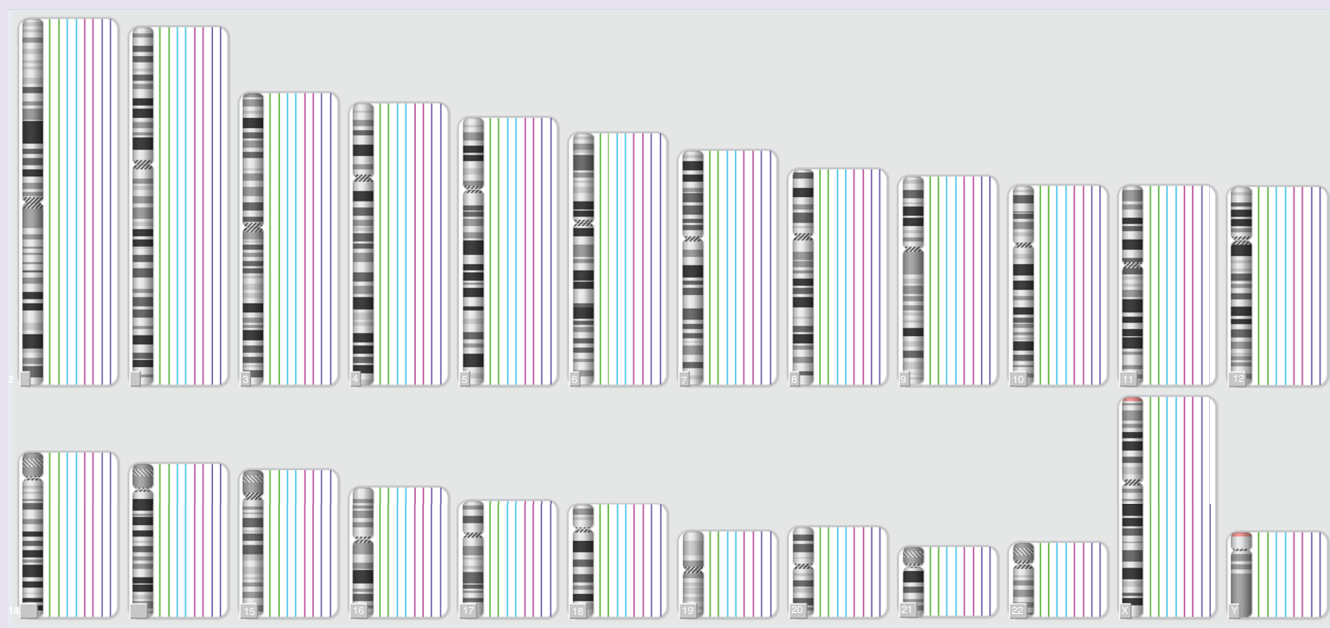

HDFa XF5, CFa923-C2, CFa923-C3, CFa923-C4

(C)

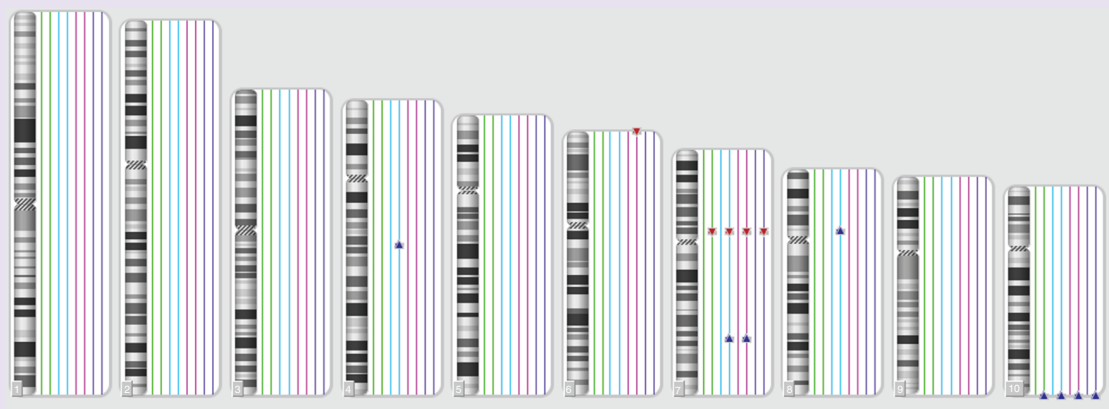

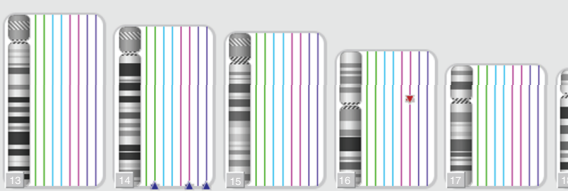

HDFa XF5, CFa923-C2, CFa923-C3, CFa923-C4

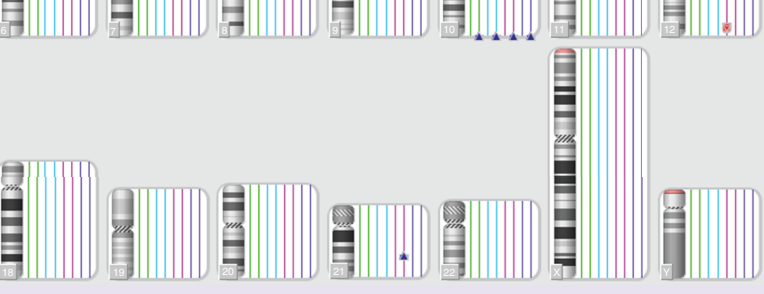

Setting: High resolution

For gain and loss: Size: 100 kb, Marker count: 50

Figure 4. Analysis of genomic integrity. (A) G-banding was performed on two of the iPSC clones to independently confirm genetic integrity. (B) KaryoStat and (C) Karyostat HD array-based whole genome hybridizations were used to analyze the genomic integrity of clones, as compared with the parental HDFa. HDFa: Adult human dermal fibroblast; iPSC: Induced pluripotent stem cell. 


\begin{tabular}{|c|c|c|c|c|c|c|}
\hline Sample & $\mathrm{Chr}$ & Size (kbp) & Gene count & Genes & CN state & Type \\
\hline CFa923-C3 & 12 & 5533 & 21 & $\begin{array}{l}\text { TMEM132B, LINC00939, LOC100128554, LINC00944, LINC00943, } \\
\text { LOC440117, FLJ37505, LINCO0507, TMEM132C, MIR3612, } \\
\text { SLC15A4, GLT1D1, TMEM132D, LOC100190940, FZD10-AS1, } \\
\text { FZD10, PIWIL1, RIMBP2, STX2, RAN, GPR133 }\end{array}$ & 1.73 & LossMosaic \\
\hline
\end{tabular}

Table 3A. Hotspot variant detection using ion torrent oncopanel.

\begin{tabular}{|lll|}
\hline Sample & Variants & Hot spot variants \\
\hline HDFa XF5 & 182 & 0 \\
\hline CFa923-C2 & 180 & 0 \\
\hline CFa923-C3 & 181 & 0 \\
\hline CFa923-C4 & 181 & 0 \\
\hline AOHC & 288 & 37 \\
\hline NTC & 0 & 0 \\
\hline $\begin{array}{l}\text { Summary of total variants and known hotspot variants including TP53 by oncomine comprehensive assay v3 assay. } \\
\text { HDFa: Adult human dermal fibroblast. }\end{array}$ & \\
\hline
\end{tabular}

microarray with a combination of nonpolymorphic probes (copy number $[\mathrm{CN}]$ probe) and polymorphic probes (single nucleotide polymorphism [SNP] probes) that can detect chromosomal aberrations at a resolution of $2 \mathrm{MB}$ and $50 \mathrm{~kb}$, respectively. Results from KaryoStat represented as KaryoView did not reveal any blue triangles that represent chromosomal gains or red triangles that represent chromosomal loss, indicating absence of chromosomal gain or loss in the parental fibroblast and the three iPSC clones (Figure 4B). The same samples, when analyzed using the higher resolution KaryoStat HD, showed presence of subchromosomal loss or gain at multiple loci (Figure 4C). For example, the parental fibroblast exhibited chromosomal loss of p12.1 on chromosome 7 and chromosomal gains of q26.3 and q32.33 on chromosome 10 and 14, respectively. iPSC clones displayed a number of additional subchromosomal gains and losses along with those that were present in the parental line. Specifically, the CFa923-C3 clone displayed mosaic-loss of q24.32 on Chr12. A detailed list of chromosomal aberrations is included in Supplementary Table 2. However, upon alignment of these chromosomal aberrations with known array artifacts, only the mosaic-loss on chromosome 12 of CFa923-C3 clone was confirmed. Table 2 provides a detailed description of the affected chromosome segment and lists the affected genes. None of the affected genes were associated with any known disease phenotype or have any known major impact in the behavior of the iPSC clones.

To further ensure that the iPSC clones were devoid of TP53 and other variants associated with known human cancers, ion torrent oncomine comprehensive assay v3 (OCAv3) was performed. This assay is a NGS-based multiple-biomarker assay that detects relevant single nucleotide variants, copy number variants, gene fusions and indels from 161 unique cancer driver genes including increased kinase domain coverage and representation of genes involved in DNA repair. Results from this assay, represented in Table 3A, indicates a comparable number of variants between parental fibroblast (182) and iPSCs (180-182), with an absence of variants that are associated with cancer hotspots. Further examination of TP53 revealed that the variant remains the same across parental and iPSCs lines (Table 3B).

Finally, iPSC lines were authenticated by comparing the short tandem repeat (STR) profiles of the iPSC clones with the parental fibroblast using the Identifiler Direct PCR Amplification Kit that amplifies 15 unique STR loci and the amelogenin gender-determining markers. Apart from the parental line and the iPSC clones, two other cell lines - H9 ESC and a Gibco iPSC - were included in this assay. Results confirm that three iPSC clones (CFa923-C2, CFa923-C3 and CFa923-C4) match with the parental fibroblast (HDFa XF5), but differ from both the Gibco iPSC and H9 ESC lines (Table 4). Overall, these results suggest that iPSC clones generated using CTS CytoTune 2.1, are of good quality, able to differentiate into all three germ layers, and preserved genetic and genotypic identity.

\section{Discussion}

Pluripotent stem cells hold immense therapeutic potential; however, the generation of clinical grade cells banks suitable for translational use still remains a challenge [47]. A critical need is the availability of raw materials utilized in the overall process that includes integration-free reprogramming systems, culture media and reagents 


\begin{tabular}{|c|c|c|c|c|c|c|c|c|c|c|}
\hline Gene ID & Chrom. & Position & Ref. & Variant & Frequency & Type & Allele source & $\begin{array}{l}\text { Relative read } \\
\text { quality }\end{array}$ & Location & Allele name \\
\hline TP53 & chr17 & 7578479 & G & A & 0 & SNP & Hotspot & 195.252 & chr17:007578479 & COSM10905 \\
\hline TP53 & chr17 & 7578479 & G & C & 0 & SNP & Hotspot & 197.389 & chr17:007578479 & COSM44944 \\
\hline TP53 & chr17 & 7578479 & G & T & 0 & SNP & Hotspot & 47.3721 & chr17:007578479 & COSM43911 \\
\hline TP53 & chr17 & 7577538 & C & A & 0 & SNP & Hotspot & 117.115 & chr17:007577538 & CosM6549 \\
\hline TP53 & chr17 & 7577538 & c & G & 0 & SNP & Hotspot & 58.9882 & chr17:007577538 & COSM11491 \\
\hline TP53 & chr17 & 7577538 & c & T & 0 & SNP & Hotspot & 350.486 & chr17:007577538 & COSM10662 \\
\hline TP53 & chr17 & 7577548 & c & A & 0 & SNP & Hotspot & 136.78 & chr17:007577548 & COSM11081 \\
\hline TP53 & chr17 & 7577548 & C & G & 0 & SNP & Hotspot & 68.4572 & chr17:007577548 & COSM10957 \\
\hline TP53 & chr17 & 7577548 & c & $\mathrm{T}$ & 0 & SNP & Hotspot & 138.877 & chr17:007577548 & CosM6932 \\
\hline TP53 & chr17 & 7577539 & G & A & 0 & SNP & Hotspot & 174.184 & chr17:007577539 & COSM10656 \\
\hline TP53 & chr17 & 7577539 & G & c & 0 & SNP & Hotspot & 63.1324 & chr17:007577539 & COSM11564 \\
\hline TP53 & chr17 & 7577121 & G & A & 0 & SNP & Hotspot & 385.272 & chr17:007577121 & COSM10659 \\
\hline TP53 & chr17 & 7577121 & G & c & 0 & SNP & Hotspot & 128.015 & chr17:007577121 & COSM43843 \\
\hline TP53 & chr17 & 7577121 & G & $\mathrm{T}$ & 0 & SNP & Hotspot & 70.3962 & chr17:007577121 & COSM43909 \\
\hline TP53 & chr17 & 7577570 & C & A & 0 & SNP & Hotspot & 245.849 & chr17:007577570 & COSM11063 \\
\hline TP53 & chr17 & 7577570 & C & G & 0 & SNP & Hotspot & 253.253 & chr17:007577570 & COSM44415 \\
\hline TP53 & chr17 & 7577570 & c & $\mathrm{T}$ & 0 & SNP & Hotspot & 57.8624 & chr17:007577570 & COSM10834 \\
\hline TP53 & chr17 & 7577120 & c & A & 0 & SNP & Hotspot & 77.2036 & chr17:007577120 & COSM10779 \\
\hline TP53 & chr17 & 7577120 & C & G & 0 & SNP & Hotspot & 76.204 & chr17:007577120 & cosM43896 \\
\hline TP53 & chr17 & 7577120 & c & T & 0 & SNP & Hotspot & 416.4 & chr17:007577120 & $\cos M 10660$ \\
\hline
\end{tabular}

\begin{tabular}{|c|c|c|c|c|c|c|}
\hline & Gibco iPSC & H9 ESC & HDFa XF5 & CFa923-C2 & CFa923-C3 & CFa923-C4 \\
\hline CSF1PO & 11,11 & 11,11 & 12,13 & 12,13 & 12,13 & 12,13 \\
\hline D13S317 & 9,11 & 9,9 & 11,12 & 11,12 & 11,12 & 11,12 \\
\hline D16S539 & 11,13 & 12,13 & 11,11 & 11,11 & 11,11 & 11,11 \\
\hline D19S433 & 12,14 & 12,15 & 13,14 & 13,14 & 13,14 & 13,14 \\
\hline D21S11 & 29,29 & 30,30 & $30,33.2$ & $30,33.2$ & $30,33.2$ & $30,33.2$ \\
\hline D2S1338 & 17,24 & 18,24 & 16,23 & 16,23 & 16,23 & 16,23 \\
\hline D3S1358 & 15,18 & 13,16 & 16,17 & 16,17 & 16,17 & 16,17 \\
\hline D5S818 & 11,12 & 11,12 & 11,11 & 11,11 & 11,11 & 11,11 \\
\hline TPOX & 8,8 & 10,11 & 8,8 & 8,8 & 8,8 & 8,8 \\
\hline vWA & 17,18 & 17,17 & 15,17 & 15,17 & 15,17 & 15,17 \\
\hline
\end{tabular}

Genomic DNA was PCR amplified and resolved by capillary electrophoresis on a SeqStudio Genetic Analyzer. Electropherogram was analyzed using an automated genotyping software, GeneMapper ID. iPSC clones CFa923-C2, C3 and C4 matched exact repeat numbers at select loci with HDFa-XF5 confirming that the iPSC clones preserved genotypic identity, whereas Gibco iPSC and H9 ESC displayed varied repeat numbers profile.

iPSC: Induced pluripotent stem cell.

free of xenogeneic material, and developed under a GMP-compliant manufacturing protocols. ESC and iPSC have been generated either under fully GMP-conditions [48], or have been reported as qualified for GMP by additional testing [49-51]. Here, we report the first commercial product that supports generation and expansion 
of iPSC in xeno-free workflows from both fibroblast and blood cells. Replacement of FBS in fibroblast growth media with xeno-free components had an effect on growth rate and transduction efficiency which correlated with reprogramming efficiencies. The various media formulations tested had to be screened for overall reprogramming efficiency since systems that only supported growth or transduction efficiency were not optimal for reprogramming. The formulation that did support consistent reprogramming had a lower efficiency in comparison with FBScontaining media. Another contributing factor to reduced reprogramming efficiency was the replacement of $c-M y c$ with $L-M y c$. While CytoTune 2.1 with $L-M y c$ showed decreased reprogramming in comparison with CytoTune 2.0 with $c-M y c$, replacement of $c-M y c$ with $L-M y c$ in CytoTune 2.0 showed comparable reprogramming with CytoTune 2.1 , suggesting the reduction is mainly due to replacement of $c-M y c$ with $L-M y c$ and not due to culture media or viral formulation. In the case of $\mathrm{CD} 34^{+}$and T cells, the culture media were already xeno-free and the difference in reprogramming efficiency between CytoTune 2.0 and CTS CytoTune 2.1 can primarily be attributed to replacement of $c-M y c$ with L-Myc. Interestingly, contrary to this observation, earlier reports with episomal vectors and retrovirus indicate $L-M y c$ to show equivalent or higher reprogramming efficiency compared with c-Myc [32]. It is possible that a different stoichiometry of $L-M y c$ could be optimal for Sendai virus-based reprogramming. The reduction in reprogramming efficiency however had no impact on consistency of reprogramming across different donor cells. Further methods that improve reprogramming efficiency can be used such as addition of human serum [17] or small molecules [52].

Another critical part of generating high-quality iPSCs is comprehensive and standardized methods for characterization. This is particularly important due to the diverse genetic backgrounds, various methods and culture conditions that add to layers of complexity [53,54]. Hence, there is an imperative need for accurate and scalable high-throughput characterization methods that offer a high degree of specificity and sensitivity [40,54]. Current methods rely on a combination of in vitro and in vivo analyses based on the expression of self-renewal markers and the ability of the cells to differentiate into cell types representative of the three germ layers as a confirmation of functional pluripotency [54,55]. These methods, though informative and extensively used, are not ideal for parallel analyses of large numbers of samples and are not as amenable to high-throughput environments as molecular assays $[19,41,42]$. In a recent study which used PluriTest to confirm global molecular signature and hPSC ScoreCard for functional pluripotency, the authors proposed the combination of the two assays for routine characterization of hPSCs used for in vitro disease modeling and drug testing [56].

The importance of assessing genomic integrity and absence of variants that are recurrently observed in PSC [57] could pose a challenge in their therapeutic application [58]. Earlier studies have indicated recurrent aberrations in PSC such as gains in chromosomes 1, 12, 17, 20 and X [59] with further amplification of a region of the long arm of chromosome 20 [60]. Here, global array-based methods that can detect chromosomal gains/losses and mosaicism can be used to detect these occurring chromosome losses and gains. Even though this method does not detect balanced translocations or single nucleotide variations, it is less of an issue since these aberrations are more often observed in mouse cells and infrequent in human cells. KaryoStat and KaryoStat HD provide solutions at two different resolutions enabling easy transition from basic research to translational applications. Any chromosomal anomalies observed can be further analyzed to determine the list of genes in the affected region.

Clinical trials utilizing PSC-derived products so far have noted no tumor formation, but as more clinical trials are initiated, more detailed information on the tumorigenic potential of PSC will emerge [61]. In the meantime, in order to further establish safety, analysis of variants associated with known cancers can be carried out using a focused NGS panel. Recent studies indicate that a substantial fraction of PSC harbor TP53 mutations and 15 instances of TP53 was observed with four mutations commonly seen in cells from different labs (P151S, R181H, R248Q and R267W) [62]. This study highlights the need for careful genetic characterization of PSCs and their differentiated cell products prior to use in clinical trials. Results shown here demonstrate the absence of cancer hotspot variants including TP53 in iPSC generated using CytoTune 2.1. The total number of variants between the parental fibroblast and resulting iPSCs were similar, indicating a robust and safe workflow. Finally, cell line mix-up or cross-contamination with other cell types is a widely acknowledged issue [63], thus requiring authentication of the iPSC clones to the parental somatic cells.

\section{Conclusion}

Development of validated reagents that are compatible with workflows and integrated with comprehensive characterization tools supports process control and facilitates easy transition from research to translational applications. 


\section{Translational perspective}

As iPSC-derived cells move toward translational applications, there is a clear need to move from research-grade reagents, tools and technologies toward more regulatory friendly ones. Incorporating pre-qualified raw materials and reagents into manufacturing processes and setting up reliable and scalable supply chains is key to ensure timely manufacture of safe, and hopefully efficacious clinical products. Development of solutions and workflows that are free of animal origin components and manufactured under certified quality management systems provide the traceability required by regulatory bodies and ensure consistent performance with minimal lot-to-lot variability. Analytical tools and methods with appropriate standards help determine the quality of the intermediate master cell bank and final cell product, further mitigating safety and quality risks. This study reports the development of pre-qualified reagents and workflows combined with comprehensive characterization tools that begin to address these needs. Commercial availability of such solutions can aid with development of harmonized international standards that allow successful translation through discovery, process development and commercial phases of cell therapy product development.

\section{Summary points}

- The CTS CytoTune-iPS 2.1 kit was used to reprogram different cell types, including fibroblasts, T cells and CD34 cells. This kit is designed for use in clinical and translational research.

- Manufactured in accordance with Good Manufacturing Practice requirements.

- Completely free of animal origin components.

- Replacement of potentially oncogenic c-Myc with L-Myc.

- A xeno-free medium was identified that supports the isolation, expansion and reprogramming of donor fibroblast cells.

- An array of molecular methods were employed to characterize xeno-free derived induced pluripotent stem cell (iPSC) clones:

- Pluripotency was confirmed via an array-based global transcriptome assay, PluriTest.

- Trilineage differentiation potential was confirmed by the TaqMan hPSC Scorecard assay.

- Preservation of genomic integrity was confirmed by a high-resolution whole genome array, KaryoStat and KaryoStat HD.

- iPSC clones were further scrutinized for the occurrence of any known hotspot cancer-causing mutations that may appear from prolonged culture condition by next generation sequencing-based oncomine comprehensive assay.

- Parental identity was confirmed by matching microsatellite short tandem repeat pattern of parental cells and resulting iPSCs.

- We envision that the development of such integrated xeno-free workflows combined with comprehensive characterization offers generation of high-quality iPSC master cell banks that are suited for clinical and translational research.

Financial \& competing interests disclosure

CC MacArthur, S Pradhan, C Dargitz, M Sridharan, L Pickle, S Jackson and U Lakshmipathy are employees of Thermo Fisher Scientific. The authors have no other relevant affiliations or financial involvement with any organization or entity with a financial interest in or financial conflict with the subject matter or materials discussed in the manuscript apart from those disclosed.

No writing assistance was utilized in the production of this manuscript.

Ethical conduct of research

All human cells were purchased from commercial companies, which obtained informed consent from donors and screened the material for adventitious agents.

Supplementary material

To view the supplementary material that accompany this paper please visit the journal website at: www.futuremedicine.com/doi/s uppl/10.2217/rme-2018-0148

Open access

This work is licensed under the Attribution-NonCommercial-NoDerivatives 4.0 Unported License. To view a copy of this license, visit http://creativecommons.org/licenses/by-nc-nd/4.0/ 


\section{References}

1. Takahashi $\mathrm{K}$, Tanabe $\mathrm{K}$, Ohnuki $\mathrm{M}$ et al. Induction of pluripotent stem cells from adult human fibroblasts by defined factors. Cell 131(5), 861-872 (2007).

2. Yu J, Hu K, Smuga-Otto K et al. Human induced pluripotent stem cells free of vector and transgene sequences. Science 324(5928), 797-801 (2009).

3. Takahashi K, Yamanaka S. A decade of transcription factor-mediated reprogramming to pluripotency. Nat. Rev. Mol. Cell. Biol. 17(3), 183-193 (2016).

4. Inoue H, Nagata N, Kurokawa H, Yamanaka S. iPS cells: a game changer for future medicine. EMBO J. 33(5), 409-417 (2014).

5. Passier R, Orlova V, Mummery C. Complex tissue and disease modeling using hiPSCs. Cell Stem Cell 18(3), 309-321 (2016).

6. Okita K, Nakagawa M, Hyenjong H, Ichisaka T, Yamanaka S. Generation of mouse induced pluripotent stem cells without viral vectors. Science 322(5903), 949-953 (2008).

7. Fusaki N, Ban H, Nishiyama A, Saeki K, Hasegawa M. Efficient induction of transgene-free human pluripotent stem cells using a vector based on Sendai virus, an RNA virus that does not integrate into the host genome. Proc. Jpn. Acad. Ser. B Phys. Biol. Sci. 85(8), 348-362 (2009).

8. Warren L, Manos PD, Ahfeldt T et al. Highly efficient reprogramming to pluripotency and directed differentiation of human cells with synthetic modified mRNA. Cell Stem Cell 7(5), 618-630 (2010).

9. Anokye-Danso F, Trivedi CM, Juhr D et al. Highly efficient miRNA-mediated reprogramming of mouse and human somatic cells to pluripotency. Cell Stem Cell 8(4), 376-388 (2011).

10. Zhou H, Wu S, Joo JY et al. Generation of induced pluripotent stem cells using recombinant proteins. Cell Stem Cell 4(5), 381-384 (2009).

11. Nakagawa M, Koyanagi M, Tanabe K et al. Generation of induced pluripotent stem cells without Myc from mouse and human fibroblasts. Nat. Biotechnol. 26(1), 101-106 (2008).

12. Choi HW, Kim JS, Choi S et al. Neural stem cells differentiated from iPS cells spontaneously regain pluripotency. Stem Cells 32(10), 2596-2604 (2014).

13. Jia F, Wilson KD, Sun N et al. A nonviral minicircle vector for deriving human iPS cells. Nat. Methods 7(3), 197-199 (2010).

14. Zhou W, Freed CR. Adenoviral gene delivery can reprogram human fibroblasts to induced pluripotent stem cells. Stem Cells 27(11), 2667-2674 (2009).

15. Schlaeger TM, Daheron L, Brickler TR et al. A comparison of non-integrating reprogramming methods. Nat. Biotechnol. 33(1), 58-63 (2015).

16. Churko JM, Lee J, Ameen M et al. Transcriptomic and epigenomic differences in human induced pluripotent stem cells generated from six reprogramming methods. Nat. Biomed. Eng.1, 826-837 (2017).

17. Wiley LA, Burnight ER, Deluca AP et al. cGMP production of patient-specific iPSCs and photoreceptor precursor cells to treat retinal degenerative blindness. Sci. Rep. 6, 1-16, 30742 (2016).

18. Abeyta MJ, Clark AT, Rodriguez RT, Bodnar MS, Pera RA, Firpo MT. Unique gene expression signatures of independently-derived human embryonic stem cell lines. Hum. Mol. Genet. 13(6), 601-608 (2004).

19. Bock C, Kiskinis E, Verstappen G et al. Reference Maps of human ES and iPS cell variation enable high-throughput characterization of pluripotent cell lines. Cell 144(3), 439-452 (2011).

20. Cheng L, Hansen NF, Zhao L et al. Low incidence of DNA sequence variation in human induced pluripotent stem cells generated by nonintegrating plasmid expression. Cell Stem Cell 10(3), 337-344 (2012).

21. Draper JS, Smith K, Gokhale P et al. Recurrent gain of chromosomes $17 \mathrm{q}$ and 12 in cultured human embryonic stem cells. Nat. Biotechnol. 22(1), 53-54 (2004).

22. Enver T, Soneji S, Joshi $\mathrm{C}$ et al. Cellular differentiation hierarchies in normal and culture-adapted human embryonic stem cells. Hum. Mol. Genet. 14(21), 3129-3140 (2005).

23. Vitale AM, Matigian NA, Ravishankar S et al. Variability in the generation of induced pluripotent stem cells: importance for disease modeling. Stem Cells Transl. Med. 1(9), 641-650 (2012).

24. Pamies D, Bal-Price A, Simeonov A et al. Good cell culture practice for stem cells and stem-cell-derived models. ALTEX 34(1), 95-132 (2017)

25. Rohani L, Johnson AA, Naghsh P, Rancourt DE, Ulrich H, Holland H. Concise review: molecular cytogenetics and quality control: clinical guardians for pluripotent stem cells. Stem Cells Transl. Med. 7(12), 867-875 (2018).

26. Silva M, Daheron L, Hurley $\mathrm{H}$ et al. Generating iPSCs: translating cell reprogramming science into scalable and robust biomanufacturing strategies. Cell Stem Cell 16(1), 13-17 (2015).

27. International Stem Cell I. Assessment of established techniques to determine developmental and malignant potential of human pluripotent stem cells. Nat. Commun. 9(1), 1-15, 1925 (2018). 
28. Sullivan S, Stacey GN, Akazawa C et al. Quality control guidelines for clinical-grade human induced pluripotent stem cell lines. Regen. Med. 13(7), 859-866 (2018).

29. ISO/TS. Biotechnology. Ancillary materials present during the production of cellular therapeutic products. Best practice guidance for ancillary material users (2018). www.iso.org/standard/70983.html 20399-3:2018

30. PAS. Evaluation of materials of biological origin used in the production of cell-based medicinal products. 157, 2015 (2015). https://shop.bsigroup.com/forms/PASs/PAS-1572015

31. Okita K, Ichisaka T, Yamanaka S. Generation of germline-competent induced pluripotent stem cells. Nature 448(7151), 313-317 (2007).

32. Nakagawa M, Takizawa N, Narita M, Ichisaka T, Yamanaka S. Promotion of direct reprogramming by transformation-deficient Myc. Proc. Natl Acad. Sci. USA 107(32), 14152-14157 (2010).

33. Singh U, Quintanilla RH, Grecian S, Gee KR, Rao MS, Lakshmipathy U. Novel live alkaline phosphatase substrate for identification of pluripotent stem cells. Stem Cell Rev. 8(3), 1021-1029 (2012).

34. Fergus J, Quintanilla R, Lakshmipathy U. Characterizing pluripotent stem cells using the TaqMan ${ }^{\circledR}$ hPSC Scorecard ${ }^{\text {TM }}$ Panel. Methods Mol. Biol. 1307, 25-37 (2016).

35. Quintanilla RH Jr, Asprer JS, Vaz C, Tanavde V, Lakshmipathy U. CD44 is a negative cell surface marker for pluripotent stem cell identification during human fibroblast reprogramming. PLoS ONE 9(1), 1-10, e85419 (2014).

36. Bulic-Jakus F, Katusic Bojanac A, Juric-Lekic G, Vlahovic M, Sincic N. Teratoma: from spontaneous tumors to the pluripotency/malignancy assay. Wiley Interdiscip. Rev. Dev. Biol. 5(2), 186-209 (2016).

37. Gropp M, Shilo V, Vainer G et al. Standardization of the teratoma assay for analysis of pluripotency of human ES cells and biosafety of their differentiated progeny. PLoS ONE 7(9), 1-10, e45532 (2012).

38. Masuda $\mathrm{S}$, Yokoo $\mathrm{T}$, Sugimoto $\mathrm{N}$ et al. A simplified in vitro teratoma assay for pluripotent stem cells injected into rodent fetal organs. Cell Med. 3(1-3), 103-112 (2012).

39. Wesselschmidt RL. The teratoma assay: an in vivo assessment of pluripotency. Methods Mol. Biol. 767, 231-241 (2011).

40. Asprer JS, Lakshmipathy U. Current methods and challenges in the comprehensive characterization of human pluripotent stem cells. Stem Cell Rev. 11(2), 357-372 (2015).

41. Muller FJ, Schuldt BM, Williams R et al. A bioinformatic assay for pluripotency in human cells. Nat. Methods 8(4), 315-317 (2011).

42. Tsankov AM, Akopian V, Pop R et al. A qPCR ScoreCard quantifies the differentiation potential of human pluripotent stem cells. Nat. Biotechnol. 33(11), 1182-1192 (2015).

43. Mack AA, Kroboth S, Rajesh D, Wang WB. Generation of induced pluripotent stem cells from CD34+ cells across blood drawn from multiple donors with non-integrating episomal vectors. PLoS ONE 6(11), 1-14, e27956 (2011).

44. Chen G, Gulbranson DR, Hou Z et al. Chemically defined conditions for human iPSC derivation and culture. Nat. Methods 8(5), 424-429 (2011).

45. Lee KI, Lee SY, Hwang DY. Extracellular matrix-dependent generation of integration- and xeno-free iPS cells using a modified mRNA transfection method. Stem Cells Int. 2016, 1-11, 6853081 (2016).

46. Ross PJ, Suhr ST, Rodriguez RM et al. Human-induced pluripotent stem cells produced under xeno-free conditions. Stem Cells Dev 19(8), 1221-1229 (2010).

47. Garreta E, Sanchez S, Lajara J, Montserrat N, Belmonte JCI. Roadblocks in the path of iPSC to the clinic. Curr. Transplant. Rep. 5(1), 14-18 (2018).

48. Baghbaderani BA, Tian X, Neo BH et al. cGMP-manufactured human induced pluripotent stem cells are available for pre-clinical and clinical applications. Stem Cell Rep. 5(4), 647-659 (2015).

49. Crook JM, Peura TT, Kravets L et al. The generation of six clinical-grade human embryonic stem cell lines. Cell Stem Cell 1(5), 490-494 (2007).

50. Tannenbaum SE, Turetsky TT, Singer O et al. Derivation of xeno-free and GMP-grade human embryonic stem cells-platforms for future clinical applications. PLoS ONE 7(6), 1-16, e35325 (2012).

51. Wang J, Hao J, Bai D et al. Generation of clinical-grade human induced pluripotent stem cells in Xeno-free conditions. Stem Cell Res. Ther. 6, 223 (2015).

52. Zhang Y, Li W, Laurent T, Ding S. Small molecules, big roles - the chemical manipulation of stem cell fate and somatic cell reprogramming. J. Cell. Sci. 125(Pt 23), 5609-5620 (2012).

53. Salomonis N, Dexheimer PJ, Omberg L et al. Integrated Genomic Analysis of Diverse Induced Pluripotent Stem Cells from the Progenitor Cell Biology Consortium. Stem Cell Rep. 7(1), 110-125 (2016).

54. D'antonio M, Woodruff G, Nathanson JL et al. High-throughput and cost-effective characterization of induced pluripotent stem cells. Stem Cell Rep. 8(4), 1101-1111 (2017).

55. Marti M, Mulero L, Pardo C et al. Characterization of pluripotent stem cells. Nat. Protoc. 8(2), 223-253 (2013). 
56. Bouma MJ, Van Iterson M, Janssen B, Mummery CL, Salvatori DCF, Freund C. Differentiation-defective human induced pluripotent stem cells reveal strengths and limitations of the teratoma assay and in vitro pluripotency assays. Stem Cell Rep. 8(5), 1340-1353 (2017).

57. Kilpinen H, Goncalves A, Leha A et al. Common genetic variation drives molecular heterogeneity in human iPSCs. Nature 546(7658), 370-375 (2017).

58. Yoshihara M, Hayashizaki Y, Murakawa Y. Genomic instability of iPSCs: challenges towards their clinical applications. Stem Cell Rev. 13(1), 7-16 (2017).

59. Peterson SE, Loring JF. Genomic instability in pluripotent stem cells: implications for clinical applications. J. Biol. Chem. 289(8), 4578-4584 (2014).

60. Laurent LC, Ulitsky I, Slavin I et al. Dynamic changes in the copy number of pluripotency and cell proliferation genes in human ESCs and iPSCs during reprogramming and time in culture. Cell Stem Cell 8(1), 106-118 (2011).

61. Lee AS, Tang C, Rao MS, Weissman IL, Wu JC. Tumorigenicity as a clinical hurdle for pluripotent stem cell therapies. Nat. Med. 19(8), 998-1004 (2013).

62. Merkle FT, Ghosh S, Kamitaki N et al. Human pluripotent stem cells recurrently acquire and expand dominant negative P53 mutations. Nature 545(7653), 229-233 (2017).

63. Yu M, Selvaraj SK, Liang-Chu MM et al. A resource for cell line authentication, annotation and quality control. Nature 520(7547), 307-311 (2015). 